\title{
Attentional and oculomotor capture with static singletons
}

\author{
JAN THEEUWES, GIEL-JAN DE VRIES, and RICHARD GODIJN \\ Vrije Universiteit, Amsterdam, The Netherlands
}

\begin{abstract}
Previous research has shown that in visual search static singletons have the ability to capture attention (Theeuwes, 1991a, 1992). The present study investigated whether these singletons also have the ability to capture the eyes. Participants had to make an eye movement and respond manually to a shape singleton while a color singleton was present. When participants searched for a unique shape while a unique color singleton was present there was strong attentional and oculomotor capture (Experiment 1). However, when participants searched for a specific-shape singleton (a green circle) when a specific-color singleton (a red element) had to be ignored, there was attentional capture but no oculomotor capture (Experiment 2). The results suggest that an attentional set for a specific feature value defining both the target and the distractor (as in Experiment 2) allows such a fast disengagement of attention from the location of the distractor that a saccade execution to that location is prevented.
\end{abstract}

A crucial question is the extent to which we are able to exert control over what we select from the visual environment. Overt or covert selection may be controlled by the properties of the stimulus field or by the intentions, goals, and beliefs of the observer (for reviews, see, e.g., Egeth \& Yantis, 1997; Ruz \& Lupiáñez, 2002; Theeuwes, 1993; Theeuwes \& Godijn, 2001; Yantis, 2000). When we intentionally select only those objects and events needed for our current tasks, selection is said to occur in a voluntary, goal-directed manner. When, irrespective of our goals and beliefs, specific properties present in the visual field determine what we select, this selection is said to occur in an involuntary, stimulus-driven manner. When objects or events receive priority independent of the observer's goals and beliefs, one refers to attentional capture when such an event or object captures only our attention (e.g., Yantis, 1996), and one refers to oculomotor capture when such an event triggers an exogenous saccade to the location of the object or event (Theeuwes, Kramer, Hahn, \& Irwin, 1998; Theeuwes, Kramer, Hahn, Irwin, \& Zelinsky, 1999).

To investigate whether salient singletons capture attention in a purely stimulus-driven manner, Theeuwes (1991a, 1992, 1994) developed a paradigm referred to as the irrelevant singleton (Yantis \& Egeth, 1999) or the additional singleton paradigm (e.g., Simons, 2000). For example, Theeuwes (1991a) investigated whether it was possible to selectively attend to a unique element within a particular stimulus dimension such as color, shape, or brightness. Theeuwes (1991a, Experiment 2) presented

We thank Steven Yantis, Roger Remington, and Kimron Shapiro for their valuable comments. Correspondence should be addressed to J. Theeuwes, Department of Cognitive Psychology, Vrije Universiteit, Van der Boechorststraat 1, 1081 BT Amsterdam, The Netherlands (e-mail: j.theeuwes@psy.vu.nl). participants with displays consisting of colored circles or diamonds appearing on the circumference of an imaginary circle. Participants had to search for an element that was unique within a particular dimension. For example, in the shape condition participants consistently searched for a target element that was unique within the shape dimension (i.e., a circle between diamonds or a diamond between circles). In other words, the target element popped out within the shape dimension. Line segments of different orientations appeared in the circles and diamonds. Participants had to determine the orientation of the line segment appearing in the unique shape. Time to find the shape singleton increased when an irrelevantcolor singleton was present (i.e., one of the circles was red). These results indicated that an item unique in a task-irrelevant dimension can interfere with search for an item unique in a task-relevant dimension. Thus, during a search for a unique shape (a diamond between circles or a circle between diamonds), a unique color (a red between green elements or a green between red elements) interfered. This indicated that it was impossible to selectively attend to a particular dimension without interference from another, irrelevant, dimension.

Theeuwes (1992) elaborated these results by showing that even when the exact target and distractor features were known, complete selectivity was not possible. For example, in Experiment 2 of Theeuwes (1992), participants searched consistently for a green circle surrounded by green diamonds. In the control condition all the shapes had the same color. In the distractor condition, one shape had a unique color (e.g., red). Time to find the green circle was consistently longer when an irrelevant-color singleton was present, suggesting that participants could not ignore the color singleton. Furthermore, in both Theeuwes (1991a) and Theeuwes (1992) it was shown that selectivity depended on the relative salience of the stimulus 
attributes: When the color singleton was made less salient (by reducing the color difference between the target and the nontarget elements) than the shape singleton, the shape singleton interfered with search for the color singleton and the color singleton no longer interfered with the search for the shape singleton. Theeuwes (1991a, 1992, 1994, 1996) explained the increase in search time in conditions in which an irrelevant singleton was present in terms of attentional capture. Because the irrelevant singleton exogenously captured attention, it required more time before a response could be emitted. It was suggested that given the observation that selectivity completely depended on the relative salience of the singleton target and distractor, early visual processing is driven exclusively by bottom-up factors such as salience. It was further argued that irrespective of the attentional set of the observer, spatial attention is automatically and involuntarily captured by the most salient singleton. The shift of spatial attention to the location of the singleton implies that the singleton is selected for further processing. If this singleton is the target, a response is made. If it is not the target, attention is directed to the next most salient singleton. The initial shift of attention to the most salient singleton is thought to be the result of relatively inflexible, "hardwired" mechanisms that are triggered by the presence of feature difference signal interrupts. It is assumed that at each location in the visual field, a local feature contrast is calculated that represents how different that object is within a particular primitive feature dimension (color, shape, contrast, etc.). The notion is similar to that of Koch and Ullman (1985), who introduced the concept of a salience map to accomplish preattentive early selection. This two-dimensional map encodes the salience of objects in their visual environment. Neurons in this map compete with each other, giving rise to a single winning location (cf. winner-take-all) that contains the most salient element. If this location is inhibited, the next salient location will receive spatial attention (see also Itti \& Koch, 2000; Nothdurft, 2000; Sagi \& Julesz, 1985).

Theeuwes's (1991a, 1992, 1994) claim that attentional capture is automatic, relatively inflexible, and hardwired was challenged by Bacon and Egeth (1994). They basically replicated Theeuwes's (1992) shape singleton experiment, in which a color singleton interfered with search. However, in one of their experiments, the target shape was no longer unique because additional shapes (i.e., squares and triangles) were added to the display. They showed that in this condition the color singleton no longer interfered. They argued that adding additional shapes eliminated the uniqueness of the target, and therefore observers could not simply respond to "uniqueness" to find the target. Bacon and Egeth suggested that under these conditions, in which participants could no longer rely on a difference signal detection (referred to as singleton detection mode), they switched strategies and relied on the so-called feature detection mode. In a feature detection mode, observers are able to exclusively direct their at- tention to the relevant feature, and irrelevant singletons no longer interfere. Bacon and Egeth concluded that "goal-directed selection of a specific known featural singleton identity may override stimulus-driven capture by salient singletons" (p. 493). These results suggest that when observers "choose" a feature singleton detection mode, attentional capture by irrelevant singletons can be eliminated. The notion that choosing a search strategy allows attentional control suggests that attentional capture is to some extent under top-down control.

The notion that attentional capture is under top-down control fits very well with the contingent capture hypothesis suggested by Folk and colleagues (Folk \& Remington, 1998; Folk, Remington, \& Johnston, 1992; Folk, Remington, \& Wright, 1994). On the basis of a spatial cuing paradigm (which is quite different from a visual search paradigm; see Theeuwes \& Godijn, 2001, for a discussion), they argued that there is never bottom-up capture of attention. Instead they argued that the ability of a singleton to capture attention is completely contingent on whether an attentional-capturing stimulus is consistent with top-down settings, which are established "offline" on the basis of current attentional goals. According to the contingent capture model, only stimuli that match the top-down control settings will capture attention; stimuli that do not match the top-down settings will be ignored. Thus according to this theory, top-down control is possible even when target and distractor are both salient singletons.

Recently, however, Theeuwes (in press) showed that the notion of singleton and feature search modes may be incorrect. As noted, Bacon and Egeth (1994) claimed that color singletons do not interfere with search for a shape singleton when instead of using a singleton detection mode participants are forced to use a feature search mode. Bacon and Egeth induced a feature search mode by adding shape singletons to the display so that observers could not simply respond to "uniqueness" to find the target. Theeuwes (in press) did the same thing but used larger display sizes to ensure that the target and distractor singletons would remain salient. The results showed that under these conditions, an irrelevant-color singleton interfered with search for a shape singleton. These data show that the evidence for the existence of these differential search modes may not be as strong as typically assumed.

The dispute over whether attentional capture is genuinely bottom-up still continues (see Ruz \& Lupiáñez, 2002; Theeuwes, in press; Theeuwes \& Godijn, 2001, 2002). Meanwhile, Theeuwes and colleagues (Theeuwes et al., 1998; Theeuwes et al., 1999) recently asked the question whether irrelevant singletons can capture the eyes. The so-called oculomotor capture paradigm they developed was very similar to the additional singleton paradigm. Participants had the explicit instruction to make an eye movement to the uniquely colored circle in the display. A saccade to the color singleton target was necessary because participants had to identify the letter inside the color singleton. Because the letter inside the 
color singleton was so small, its identity could be revealed only after it was fixated. In the oculomotor capture paradigm, observers had the top-down goal to rapidly make a saccade to the color singleton and respond to the letter located inside the color singleton. On some trials, a new object, presented with abrupt onset, was added to the display. Participants knew that the onset was irrelevant and also knew that they had to ignore it. The condition in which a to-be-ignored onset (the new object) was presented somewhere in the visual field was compared with a control condition in which there was no onset added to the display. The results showed that when no onset was added to the display, observers made saccades that generally went directly to the uniquely colored circle. However, in about $30 \%$ to $40 \%$ of the trials in which an onset was added to the display, the eye went in the direction of the onset, stopped briefly, and then went on to the target. The results from the oculomotor capture paradigm suggest that an irrelevant singleton-in our case a new object presented with abrupt onset-not only captures attention (see Theeuwes et al., 1999, Experiment 2; Godijn \& Theeuwes, 2002b) but also can capture the eyes. Even though observers had a top-down goal to make a saccade to the uniquely colored singleton, they could not prevent their eyes from moving to the abrupt onset in about $30 \%$ to $40 \%$ of the trials.

The observation that abrupt onsets may not only capture attention but may also capture our eyes is not surprising given the fact that there is a close relationship between the attentional and oculomotor systems (e.g., Rizzolatti, Riggio, Dascola, \& Umiltà, 1987). It is generally agreed that there is an obligatory and selective coupling between saccade execution and visual attention to one common target object (see, e.g., Deubel \& Schneider, 1996; Godijn \& Pratt, 2002; Godijn \& Theeuwes, 2002b; Hoffman \& Subramaniam, 1995). When a saccade is executed, attention precedes the eyes to the saccade target location.

One might argue that oculomotor capture occurred only in Theeuwes et al. (1998; Theeuwes et al., 1999; see also Irwin, Colcombe, Kramer, \& Hahn, 2000; Kramer, Hahn, Irwin, \& Theeuwes, 1999, 2000; Godijn \& Theeuwes, 2002a, 2002b) because the to-be-ignored singleton was an abrupt onset. There is ample evidence that visual transients have the ability to capture attention exogenously (see, e.g., Breitmeyer \& Ganz, 1976; Remington, Johnston, \& Yantis, 1992; Theeuwes, 1991b, 1994, 1995; Yantis \& Jonides, 1984). According to this view, an abrupt luminance change (i.e., increment or decrement) at a retinal location is detected by a specialized visual mechanism that is sensitive to transient change; these then signal to higher centers that an important event has occurred. ${ }^{1}$ In addition to capturing attention, an abrupt onset also will result in the activation of an eye movement program to the location of the onset (e.g., Rafal, Egly, \& Rhodes, 1994; Taylor \& Klein, 1998). There is evidence that onset transients have direct input to the superior colliculus (SC). The SC is a midbrain neural struc- ture that receives convergent afferents from different cortical and subcortical visual and cognitive centers related to eye movement control (see Munoz \& Istvan, 1998; Sparks \& Hartwich-Young, 1989). The SC sends projections to the brainstem premotor area to trigger saccadic eye movements. Because onset transients directly generate activity in the SC (within $70 \mathrm{msec}$ after display onset; see Trappenberg, Dorris, Munoz, \& Klein, 2001) it may not be surprising that in Theeuwes et al. (1998; Theeuwes et al., 1999) a large proportion of the saccades went to the onset.

The present paper addressed the question whether irrelevant static singletons that Theeuwes (1991a, 1992) has demonstrated to capture attention exogenously also have the ability to capture the eyes. Note that Theeuwes (1991 a, 1992) manipulated the salience of the irrelevantcolor singletons, and he demonstrated that these singletons were salient enough to capture attention. The same singletons were used in the present study. In addition, the singletons were presented without luminance changes to ensure that there were no transient properties that could directly trigger activity in the SC. Experiment 1 used an oculomotor version of the earlier discussed Theeuwes (1991a) additional singleton paradigm, in which observers have to search for a singleton unique within one particular stimulus dimension (search for a unique shape or search for a unique color) and ignore a singleton in the other, irrelevant, stimulus dimension. Experiment 2 used an oculomotor version of the procedure used in Theeuwes (1992) in which observers consistently searched for a particular stimulus feature and ignored another feature singleton.

In addition to measuring manual reaction time (RT) to examine whether or not the presence of the irrelevant feature slowed responding, we also measured the actual path of the eye through visual space. We expected that in line with Theeuwes (1991a, 1992), the presence of an irrelevant salient singleton would increase manual RT. In addition, we expected that if the static irrelevant singleton is able to capture the eyes, in a number of trials the eyes will move toward the irrelevant singleton before they move on to the singleton target. If, however, a transient change is necessary to trigger a saccade, the presence of the irrelevant static singleton distractor will cause attentional capture but not oculomotor capture. In that case we would expect an effect of the presence of the distractor on manual RT but no effect on the saccade path.

\section{EXPERIMENT 1}

Experiment 1 was closely modeled after Theeuwes (1991a), which addressed the selectivity between stimulus dimensions. In Theeuwes (1991a), participants responded to the orientation of a line segment located inside the singleton target. In that study, it was ensured that the line segment inside the singleton target was large enough that its orientation could be determined by directing spatial attention to that location. In the present 
study, we did just the opposite: Instead of line segments, we presented small letters inside the outline circles and diamonds. These letters were so small that participants needed to fixate the singleton to determine the identity of the letter therein. In other words, the letter was so small that the high acuity of the fovea was necessary to resolve the identity of the letter. In Experiment 1, observers had to make an eye movement to a singleton target that had either a unique shape (i.e., a diamond between circles or a circle between diamonds) or a unique color (a red element between green nontarget elements or a green element between red nontarget elements). Within a block of trials, participants consistently searched for a singleton that was unique within one particular dimension (either an element with a unique shape or an element with a unique color). In the no-distractor condition, the only singleton present in the visual field was the singleton target that needed to be fixated. In the distractor condition, another singleton unique in the task-irrelevant dimension was also present. We investigated whether participants could prevent making an exogenous eye movement to the singleton that was unique in the taskirrelevant dimension.

\section{Method}

Participants. Ten participants ranging in age from 17 to 23 years served as paid volunteers. All had normal or corrected-to-normal vision and reported having no color vision deficiency. Participants were naive as to the purpose of the study. Two observers were removed from statistical analysis because of high error rates in some conditions (above 10\%).

Apparatus. A Dell Optiplex GX1 personal computer with an Intel Pentium II Celeron $450 \mathrm{MHz}$ processor controlled the timing of the events and recorded RTs. The images were displayed on a Philips Brilliance (201P) 21-in. color monitor. The display's resolution was $1,280 \times 1,024$ with a true color (32-bit) palette at $75 \mathrm{~Hz}$. Eye movements were registered by means of an Eyelink tracker (SR Research Ltd). The system uses an infrared video-based tracking technology to compute the pupil center and pupil size of both eyes. The eye-tracking system has a $250-\mathrm{Hz}$ temporal resolution and a spatial resolution of better than $0.1^{\circ}$. Even though the system compensates for head movements, the observer's head was stabilized by means of a chinrest. An eye movement was considered a saccade when the velocity exceeded $35 \%$ sec or the acceleration exceeded $9,500^{\circ} / \mathrm{sec}^{2}$. On each trial, during the fixation display when participants fixated the center point, the eye position was automatically recalibrated to the center position to optimize the reliability of the eye movement measurements. When observers were well fixated on the center point, they pressed the space bar to initiate a trial. Stimuli were presented on a black background. The fixation point and target characters were presented in white $\left(97.94 \mathrm{~cd} / \mathrm{m}^{2}\right)$ on a black background $\left(0.00 \mathrm{~cd} / \mathrm{m}^{2}\right)$. The display elements consisted of outlined circles or diamonds that were either red (CIE $x, y$ chromaticity coordinates of $0.604 / 0.347$ ) or green (CIE $x, y$ chromaticity coordinates of $0.299 / 0.598$ ). Colors were matched for luminance $\left(21.32 \mathrm{~cd} / \mathrm{m}^{2}\right)$. After the trial ended, the screen was blanked with gray $\left(23.95 \mathrm{~cd} / \mathrm{m}^{2}\right)$. Each observer was tested in a sound-attenuated, dimly lit cubicle, with his/her head resting on a chinrest adjusted to a comfortable height. The computer monitor was positioned at $70 \mathrm{~cm}$ from the chinrest. The responses were made by pressing the "z" key and the "/" key of the computer keyboard.

Stimuli. The stimulus field consisted of eight elements that were equally spaced around the fixation point on an imaginary circle with a radius of $9.2^{\circ}$. The display elements were red- or green-colored outlined circles or diamonds. The dimensions of the circle-shaped element were $2.7^{\circ} \times 2.8^{\circ}$; the dimensions of the diamond-shaped element were $3.3^{\circ} \times 3.4^{\circ}$. Each element contained a $0.3^{\circ} \times 0.2^{\circ}$ white character. At the start of the experiment, observers fixated at a central white plus sign with the dimensions $0.4^{\circ} \times 0.4^{\circ}$. After $2,500 \mathrm{msec}$ the center fixation point changed into a cross sign. Simultaneously with the change of the fixation point, all elements in the stimulus array were presented. The letters inside the elements were randomly sampled without replacement from the set $\mathrm{S}, \mathrm{H}, \mathrm{E}$, $\mathrm{P}, \mathrm{F}, \mathrm{U}$, and $\mathrm{L}$. The target character, which was positioned in the singleton target (i.e., unique in shape or in color), was either a $\mathrm{C}$ or a reversed $\mathrm{C}$. The orientation of the character determined the response (i.e., press the "z" key for a $\mathrm{C}$ and the "/" key for a reversed C). Since the characters were very small and were located $9.2^{\circ}$ from the fovea, participants had to make a saccade to the singleton target in order to identify the target character. In the distractor condition, a distracting display element unique in the irrelevant dimension could appear at 3,6, 9, or 12 o'clock. The unique display element containing the target character could appear at one of the four intermediate positions. Therefore, the distracting display element could either be next to the element containing the target at $45^{\circ}$ of arc or could be positioned three elements away from the element containing the target character at $135^{\circ}$ of arc. The distracting display element was presented either clockwise from the target element (indicated by a plus sign) or counterclockwise (indicated by a minus sign). The four possible relative positions of target element and distracting element are further referred to as $-135^{\circ},-45^{\circ},+45^{\circ}$, or $+135^{\circ}$. In the remaining experimental conditions, no distracting element was present. The target element could appear at one of the four positions $(3,6,9$, or 12 o'clock). For all conditions, the stimulus field remained present for $3 \mathrm{sec}$ or until a manual response was made.

Depending on the condition, the target character $(\mathrm{C}$ or reversed C) was consistently positioned in the display element with the unique color or the display element with a unique shape. In the color condition, participants searched for the target character, which was located in the uniquely colored display element (red or green). In the no-distractor color condition, all elements had the same shape. In the color distractor condition, a distractor unique in the shape dimension (either a circle or a diamond) was also present. In the shape condition, participants searched for the target character, which was located in the uniquely shaped display element (circle or diamond). In the no-distractor shape condition, all elements had the same color. In the shape distractor condition, a distractor with a unique color (red or green) was also present. Figure 1 shows the various display configurations.

Over a block of trials, observers always searched consistently for a unique color or shape. The color (red or green) or the shape (diamond or circle) changed from trial to trial. In half of the trials, the target character was a $\mathrm{C}$; in the other half, a reversed $\mathrm{C}$, randomized within blocks. The target character appeared equally often at each of the possible target locations. The conditions were presented in separate blocks. Half of the observers started with the color condition, and the other half with the shape condition. Within these conditions, half of the observers started with the no-distractor condition followed by the distractor condition. For the other half the order was reversed.

Procedure. The experiment was divided into two sessions, both consisting of two blocks of 128 experimental trials. Each session started with a practice block of 128 trials. Before each session, the observers were informed about the upcoming conditions (search for a unique color or search for a unique shape). Before the start of the experiment, observers received written instructions and were allowed to ask the instructor questions. Observers were instructed to fixate the center fixation point (a cross) and to press the space bar in order to recalibrate the position of the eyes. The fixation point then changed into a plus sign as an indication that the positions of the eyes were recalibrated. Participants were told to make a speeded 
A

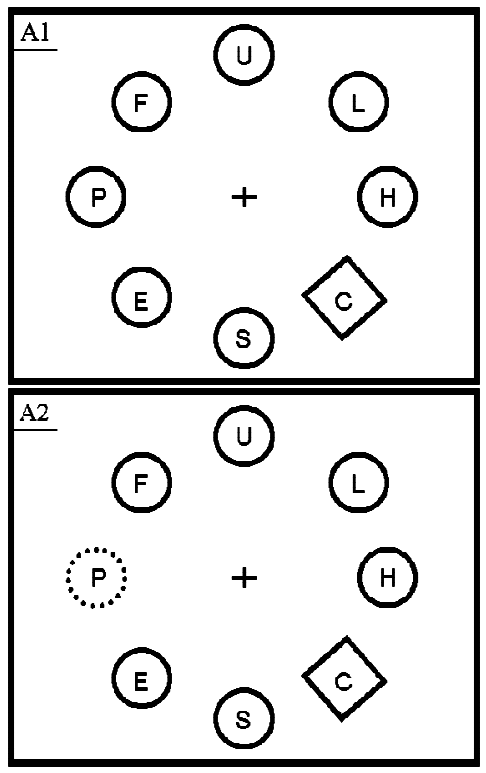

B

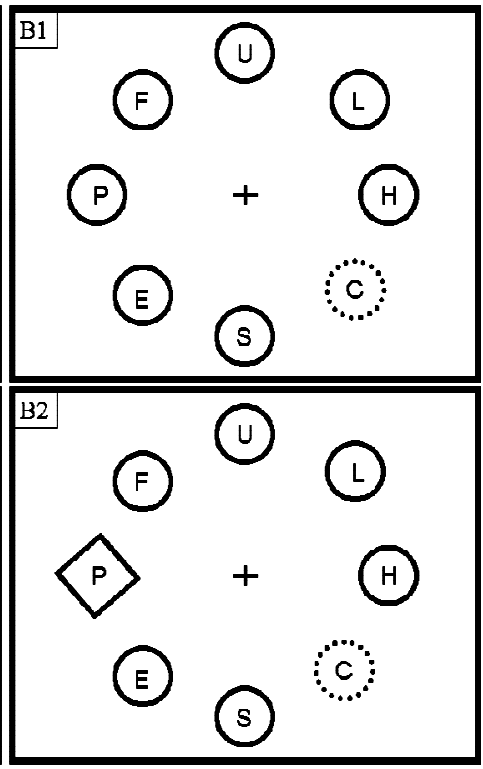

Figure 1. Examples of the stimulus display of Experiment 1. Participants searched for a unique shape (panel A) or a unique color (panel B) without a distractor (panels A1 and B1) or with a distractor (panels A2 and B2).

saccade to the element unique in the predefined dimension (color or shape) as soon as the central fixation point changed back into a cross, after 1,200 msec. Simultaneously with the modification of the fixation point, all elements were presented simultaneously on the screen. Observers were instructed to respond quickly and accurately to the character inside the singleton target by pressing the appropriate response button. A warning beep informed the participants that an error had been committed. If no response had been made after $3 \mathrm{sec}$, the trial was considered an error. After the trial finished, the display was blanked with a gray-colored background. Before the experiment the Eyelink system was calibrated. Participants had to fixate nine calibration targets that were presented randomly in a $3 \times 3$ grid across the monitor. As soon as a point was fixated, the next target was displayed on the screen.

\section{Results}

Discarded data. Saccade latencies shorter than $70 \mathrm{msec}$ (anticipation errors) and longer than $700 \mathrm{msec}$ were excluded (a loss of $1.4 \%$ of the trials). Manual RTs shorter than $100 \mathrm{msec}$ and longer than $2 \mathrm{sec}$ were excluded from the analysis, which led to a loss of $3.7 \%$ of the trials. Those trials in which participants made errors (e.g., pressing the wrong response key) were also excluded from the analyses (3.3\% of the remaining trials).

Manual RT. The first question addressed was whether the irrelevant singletons would cause a distraction effect. Manual RT, the time it took participants to press one of the response keys, was calculated for each participant. An analysis of variance (ANOVA) on mean manual RT with condition (search for a color singleton vs. search for a shape singleton) and distractor (no-distractor vs. distractor) showed a main effect of condition $[F(1,7)=552$, $p<.001]$ and of distractor $[F(1,7)=103, p<.001]$. The interaction between these factors was also reliable
$[F(1,7)=87, p<.001]$. Table 1 gives the manual RTs. As is clear from Table 1, during search for a color singleton, a shape singleton did not cause any distraction effect $[t(7)<1]$; however, during search for a shape singleton, the presence of the color singleton caused a very large distraction effect of $208 \mathrm{msec}[t(7)=11.9, p<.01]$. The results are similar to those of Theeuwes (1991a), who showed a no-distraction effect of a shape singleton during search for a color singleton and a large distraction effect of about $110 \mathrm{msec}$ caused by a color singleton during search for a shape singleton.

Saccade endpoints. The initial saccades leaving the center fixation dot were separated into saccades going directly to the target (saccades had to fall between $22.5^{\circ}$ and $-22.5^{\circ}$ of angular separation from the target) versus saccades going in other directions. In the color condition, the eyes generally went to the target (80\% to the target; $20 \%$ somewhere else in the visual field), and there was no difference between the no-distractor and distractor conditions [ $81 \%$ directly to the target in the no-distractor condition and $80 \%$ directly to the target in the distractor condition; $t(7)<1]$. In the shape condition, however, the

Table 1

Experiment 1: Manual Reaction Time (in Milliseconds) for the No-Distractor and Distractor Conditions During Search for a Unique Shape or Color

\begin{tabular}{lccc}
\hline & No-Distractor & Distractor & $\begin{array}{c}\text { Interference } \\
\text { Effect }\end{array}$ \\
\hline Search for a unique shape & 793 & 1,001 & $208 * *$ \\
Search for a unique color & 695 & 700 & 5 \\
\hline
\end{tabular}

$* * p<.01$. 
presence of the singleton distractor had a large effect on the saccade endpoint. In the no-distractor shape condition, $64 \%$ of the saccades went directly to the target. In the distractor shape condition, only $38 \%$ went directly to the target $[t(7)=4.3, p<.01]$. On a large number of trials the eyes went directly to the distractor. In fact, the eyes went as often to the distractor (38\%) as they went to the target (38\%). Overall the results indicate that during search for a shape singleton, a color singleton captured the eyes on a large proportion of trials. The results are similar to those of Theeuwes et al. (1998; Theeuwes et al., 1999), who showed that an abrupt onset caused the eyes to start moving toward the onset distractor in about $36 \%$ of the trials.

Figure 2 gives an overview of the saccade endpoints in the condition in which participants searched for a shape singleton while a color singleton was present. Figures $2 \mathrm{~A}$ and $2 \mathrm{~B}$ give the condition in which the color singleton distractor was located $45^{\circ}$ of arc to the left (Figure $2 \mathrm{~A}$ ) or to the right (Figure $2 \mathrm{~B}$ ) of the target location. Figures $2 \mathrm{C}$ and $2 \mathrm{D}$ give the condition in which the color distractor was located $135^{\circ}$ to the left (Figure 2C) or the right (Figure 2D) of the target location. These figures indicate that when the color distractor was present, the eyes often went to the location of the distractor.

Saccade latencies. Saccade latency was defined as the time that it took the eyes to start moving from the center fixation point to one of the peripheral stimuli. The timing began with the onset of the stimulus display and ended when the eyes moved away from the fixation point. In the color conditions saccade latencies toward the target were short and there was no difference in saccade latency between the no-distractor and distractor conditions (both were $169 \mathrm{msec}$ ). In the shape condition, latencies to the target in the no-distractor condition (mean of $194 \mathrm{msec}$ ) were not different from latencies to the target in the distractor condition [mean of $220 \mathrm{msec}$; $t(7)=1.71, p=.13]$. The saccade path analysis indicated that in the shape condition in which a distractor was present there were two types of eye movements: one eye movement that went directly to the target (mean latency of $220 \mathrm{msec}$ ) and one eye movement that went directly to the color singleton (mean latency of $209 \mathrm{msec}$ ). The difference in latency was not reliable.

Fixation durations at the singleton distractor. In the shape condition the eyes went to the color singleton distractor in $38 \%$ of the trials. Figure 3 shows the fixation duration after the first saccade for those saccades that went first to the distractor before they went on to the target.

The data indicate that the fixation durations were quite brief $($ mean $=151 \mathrm{msec})$. About $63 \%$ of the fixation durations were less than $150 \mathrm{msec}$, even though a large reprogramming of eye movements was required to redirect the eyes toward the singleton target. Typically it takes at least $150-250 \mathrm{msec}$ to program a completely new saccade (see Findley, 1997; Salthouse \& Ellis, 1980). These results suggest that part of the oculomotor programming was done before the eye reached the distractor location. Recent evidence suggests that spatial coding representing where a saccade should go next is conducted before the first saccade is executed (Godijn $\&$ Theeuwes, 2001). Therefore, upon arrival at the distractor location there is no need for spatial coding of the next saccade endpoint, and this results in relatively short fixation durations. Note that the fixation durations on the irrelevant-color singleton are relatively short, yet not as short as the fixation duration on an onset distractor (see Godijn \& Theeuwes, 2002a, 2002b; Theeuwes et al., 1998; Theeuwes et al., 1999). Furthermore, on some trials (about 6\%) the eyes went in the direction of the prespecified target shape, after which the eyes were redirected toward the color singleton. In these rather unusual trials in which the eyes went to the singleton distractor after fixating the singleton target, the fixation durations on the singleton target were very short (mean $=130 \mathrm{msec}$ ).

\section{Discussion}

The present findings show that static salient singletons have the ability to capture not only attention but also the eyes. In $38 \%$ of the trials the eyes went to the salient color singleton, a percentage of trials that is about the same as capture caused by abrupt onsets (which was about 35\%; see Theeuwes et al., 1999). In line with the findings of Theeuwes et al. (1998; Theeuwes et al., 1999), who used an abrupt onset as a distractor, the present experiment also shows that fixation durations at the location of the irrelevant singleton were relatively brief (about $151 \mathrm{msec}$ ), but not as brief as those reported with abrupt onsets as distractors (on average about $90 \mathrm{msec}$; see Theeuwes et al., 1999). With respect to the saccade latencies, it is important to note that when the color singleton was the target, saccade latencies were shorter (about $169 \mathrm{msec}$ ) than when the target was a shape singleton (194 msec), which confirms earlier claims that a color singleton is more salient than a shape singleton (see Theeuwes, 1991a, 1992). Unlike Theeuwes et al. (1998; Theeuwes et al., 1999), we found that the saccades that went to the distractor were not the saccades with short latencies. Indeed, latencies toward the distractor were not different from those toward the target. Obviously, onsets as used as distractors by Theeuwes et al. (1998; Theeuwes et al., 1999) have the ability to generate fast saccades, most likely because transients directly generate activity in the SC (see Godijn \& Theeuwes, 2002b).

\section{EXPERIMENT 2}

In Experiment 1, we found both attentional and oculomotor capture with static singletons. One might argue that top-down control was not possible in Experiment 1 because participants only knew the dimension to attend to (either color or shape) but not the exact feature value within that dimension. In Experiment 2, we tested this hypothesis by having participants search consistently for a green circle throughout the whole experiment. This 

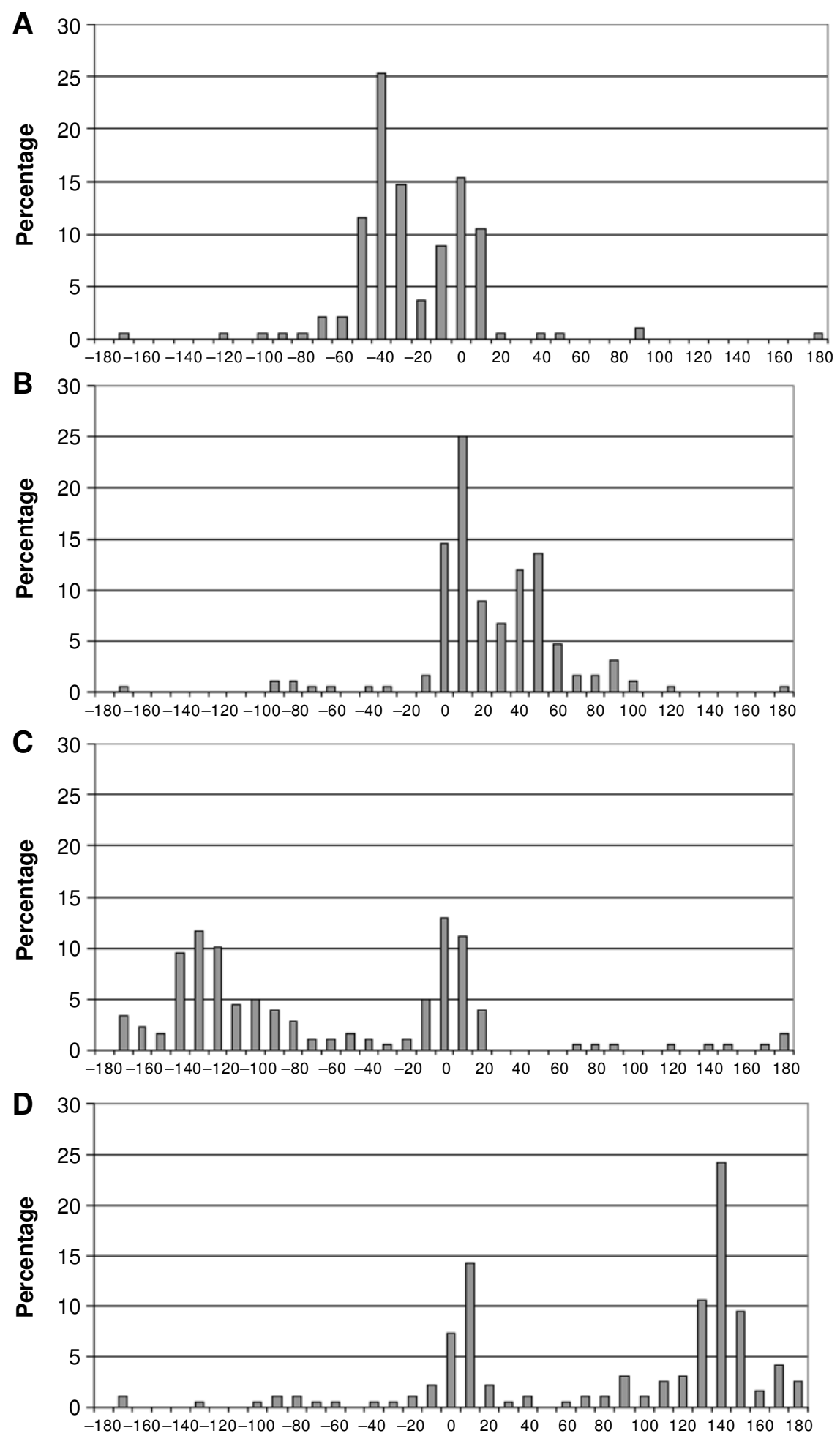

Saccade endpoints in degrees of arc from target

Figure 2. Experiment 1: Eye movement behavior when participants searched for a shape singleton while a color singleton was present at $-45^{\circ}$ of arc (panel $A$ ), $+45^{\circ}$ of arc (panel $B$ ), $-135^{\circ}$ of arc (panel C), and $+135^{\circ}$ of arc (panel D) from the target. Shown is the angle deviation of the eye from a linear path between the fixation and the target object. 


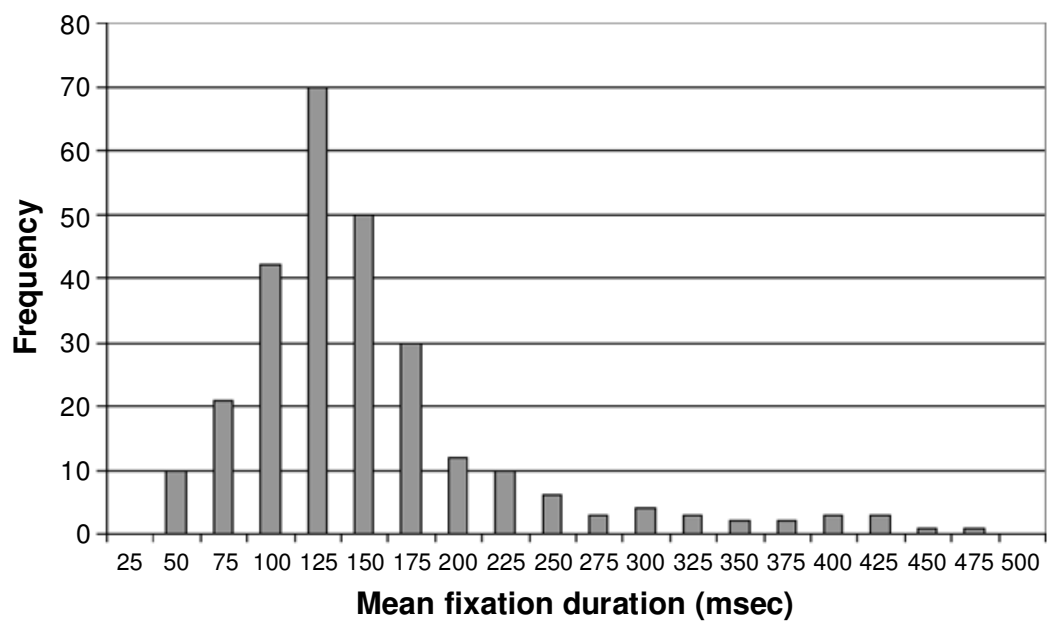

Figure 3. Experiment 1: The fixation duration after the first saccade for those saccades that went to the color singleton (distractor) and subsequently went in the direction of the shape singleton (target).

setup was identical to that of Theeuwes (1992). In the no-distractor color condition the green target circle was a color singleton; it was embedded among red circles. In the shape condition, the green target circle was a shape singleton; it was embedded among green diamonds. As in Experiment 1, participants made an eye movement toward the singleton target (i.e., the green circle) and determined the orientation of the letter $\mathrm{C}$ presented inside the singleton target. In the no-distractor condition, the only singleton present in the visual field was the green circle, which, depending on the condition, was a color or a shape singleton. In the distractor condition, another singleton in the irrelevant dimension was also present. Theeuwes (1992) showed that even when participants consistently search for a particular well-defined feature target singleton (e.g., a green circle), they cannot prevent attentional capture by an irrelevant singleton (see also Bacon \& Egeth, 1994; Experiment 1). In the present experiment, we investigated whether this attentional capture reported in Theeuwes (1992) would also result in oculomotor capture-that is, an exogenous saccade to the location of the irrelevant singleton.

\section{Method}

Participants. Sixteen participants ranging in age from 17 to 23 years served as paid volunteers. All had normal or corrected-tonormal vision and reported having no color vision def iciency. Participants were naive as to the purpose of the study.

Apparatus. The apparatus was identical to that of Experiment 1.

Stimuli. The stimulus field was identical to that of Experiment 1 except that the target was always a green circle. In the no-distractor color condition, the green circle was always surrounded by red circles. In the no-distractor shape condition, the green circle was surrounded by green diamonds. In the color distractor condition, one of the red circles was replaced by a red diamond (i.e., an irrelevantshape singleton). In the shape distractor condition, one of the green diamonds was replaced by a red diamond (i.e., an irrelevant-color singleton).

\section{Results}

Discarded data. Saccade latencies that were shorter than $70 \mathrm{msec}$ (anticipationerrors) and longer than $700 \mathrm{msec}$ were excluded (a loss of $1.7 \%$ of the trials). Manual RTs shorter than $100 \mathrm{msec}$ and longer than $2 \mathrm{sec}$ were excluded from the analysis, which led to a loss of $2.1 \%$ of the trials. Those trials in which participants made a manual error were also excluded from the analyses (a total of $3 \%)$.

Manual RT. There was a main effect of condition,indicating that searching for a color singleton was faster $($ mean $=653 \mathrm{msec})$ than searching for a shape singleton $[$ mean $=715 \mathrm{msec} ; F(1,15)=46.2, p<.01]$. In addition, there was a main effect of distractor [no-distractor mean = $677 \mathrm{msec}$ vs. distractor mean $=692 \mathrm{msec} ; F(1,15)=6.3$, $p<.05$; Table 2]. When trials in which the eyes went to the distractor singleton before they went to the target singleton were excluded from the analysis there was still a main effect of distractor on manual RT $[F(1,15)=6.1$, $p<.03$ ].

Additional planned comparisons showed that in the shape condition, participants were slower when a color distractor was present (mean RT $=725 \mathrm{msec}$ ) than when

Table 2

Experiment 2: Manual Reaction Time (in Milliseconds) for the No-Distractor and Distractor Conditions During Search for a Specific Shape Singleton (a Circle) or a Specific Color Singleton (a Green Element)

\begin{tabular}{lccc}
\hline & No-Distractor & Distractor & $\begin{array}{c}\text { Interference } \\
\text { Effect }\end{array}$ \\
\hline $\begin{array}{c}\text { Search for circle } \\
\quad \text { (shape singleton) }\end{array}$ & 705 & 725 & $20^{*}$ \\
$\begin{array}{c}\text { Search for green } \\
\text { (color singleton) }\end{array}$ & 648 & 659 & 11 \\
\hline
\end{tabular}


no distractor was present [mean $\mathrm{RT}=705 \mathrm{msec} ; t(15)=$ $2.2, p<.05]$. In the color trials there was no difference between the distractor and no-distractor conditions [mean RT of $648 \mathrm{msec}$ for the no-distractor condition vs. a mean RT of $659 \mathrm{msec}$ for the distractor condition; $t(15)=1.5$, $p=.13$ ]. These findings on manual RTs are in line with the results of Theeuwes (1992), who found a distractor effect of an irrelevant-color singleton during search for a shape singleton but no effect during search for a color singleton when a shape singleton was present. The interference effect reported (about $20 \mathrm{msec}$ ) is comparable to the interference effect reported by Theeuwes (1992).

Saccade endpoints. In the color condition the eyes generally went directly to the target (95\%), and there was no difference between the no-distractor and distractor conditions ( $95 \%$ vs. $94 \%$ ). In the shape condition, however, the presence of the distractor color singleton had an effect on the saccade endpoint. In the no-distractor shape condition, $76 \%$ of the saccades went directly to the target. In the distractor shape condition, however, only $67 \%$ went directly to the target $[t(15)=4.8, p<.001]$. These results indicate that in line with the effects on manual RT, the presence of a color singleton distractor had an effect on oculomotor behavior, whereas the presence of a shape singleton distractor had no effect.

To determine whether the irrelevant singleton caused oculomotor capture, we determined whether the eyes went to the singleton distractor. The results showed that there was almost no oculomotor capture in either the color or the shape condition. In the color condition the eyes went to the distractor in only $0.5 \%$ of the trials. In the shape condition, this percentage was somewhat higher $(1.5 \%)$. Even though the difference between these conditions was reliable $[t(15)=2.6, p<.05]$, it is hard to argue that the irrelevant singleton captured the eyes given that this occurred in only $1.5 \%$ of the trials. Even though in the shape condition the presence of a color distractor had an effect on oculomotor behavior (fewer eye movements that went directly to the target), this resulted in oculomotor capture in only $1.5 \%$ of the trials. Obviously in the shape condition the color singleton distractor caused the eyes to go to locations (in $31 \%$ of the trials) other than the location of the target or the singleton distractor.

Saccade latencies. We analyzed the saccade latencies when the eyes went directly to the target. In the color conditions the mean latency was $168 \mathrm{msec}$ for both the distractor and the no-distractor conditions. In the shape condition saccade latencies were longer than in the color conditions [mean $=184 \mathrm{msec} ; F(1,15)=20.3, p<.001$ ] and there was no difference between the distractor and no-distractor conditions. When the eyes did not go the singleton target, mean saccade latency was $164 \mathrm{msec}$. Saccade latencies toward the singleton target were longer than latencies of saccades that did not go to the target [184 msec vs. $164 \mathrm{msec} ; F(1,15)=205, p<.001]$. The results indicate that on a portion of the trials, participants made saccades with short latencies that obviously did not land at the location of the target or at the location of the singleton distractor.

\section{Discussion}

The present experiment shows that when participants searched for a color singleton the presence of a shape singleton had no effect on manual RT. During search for a shape singleton, the presence of a color singleton increased manual RT by about $20 \mathrm{msec}$, an effect that is comparable to that found by Theeuwes (1992). However, unlike Experiment 1, Experiment 2 showed that the color singleton did not cause any oculomotor capture. Even though the presence of a color singleton caused a slight increase in the number of trials in which the eyes went to the color singleton, it is clear that one cannot speak of oculomotor capture when this occurred in only $1.5 \%$ of the trials. Manual RT was greater in the color distractor condition because in a large portion of the trials (about $31 \%$ ) the eyes simply went to a location in the visual field that contained neither the target nor the distractor. The data from saccade latencies suggest that the saccades with a short latency (on average $164 \mathrm{msec}$ ) typically went in the wrong direction whereas the saccades with a relatively long latency (about $184 \mathrm{msec}$ ) went to the target. Furthermore, as in Experiment 1, saccade latencies to the color singleton target were shorter than latencies to the shape singleton target, providing evidence that a color singleton is more salient than a shape singleton. ${ }^{2}$

\section{GENERAL DISCUSSION}

The results regarding manual RT are similar to those of Theeuwes (1991a, 1992). As in Theeuwes (1991a), when participants searched for a singleton unique within the shape dimension (a diamond between circles or a circle between diamonds), a singleton unique in the color dimension (a red item between green items or a green between red items) caused a large interference effect (110 msec in Theeuwes, 1991a, and $208 \mathrm{msec}$ in the present experiment). In addition, as in Theeuwes (1992), when the to-be-searched shape singleton target and color distractor were kept constant over trials, the interference effect was strongly reduced. Yet, a reliable interference effect of about $25 \mathrm{msec}$ remained.

It is important to consider why we found strong oculomotor capture in Experiment 1 and no oculomotor capture in Experiment 2. It appears that selectivity toward a stimulus dimension such as shape or color without knowledge of the exact feature values allows much less top-down control than does knowledge of the exact value of the feature of both the target and the singleton distractor. These results are consistent with the hypothesis of Bacon and Egeth (1994), who claimed that in a singleton detection mode observers have no top-down control, whereas in a feature detection mode they are able to prevent attentional capture. Obviously in Experiment 1, in which observers knew only the dimension of the target singleton (i.e., an element with a unique color or shape but not the exact feature value), they had to rely on the singleton detection mode. As predicted by Bacon and Egeth, this should result in attentional capture. The present Experiment 1 shows that it results not only in atten- 
tional capture but also in oculomotor capture. In Experiment 2 , observers knew exactly what they were searching for (i.e., a green circle), and this allowed them to use the feature detection mode. Bacon and Egeth showed that in conditions in which participants can engage in a feature detection mode, attentional capture by irrelevant singletons is absent. Our present Experiment 2 does not show that attentional capture is absent but it does show that no oculomotor capture occurs under these circumstances. One might claim that in a feature detection mode, attentional capture is very much reduced (from 110 to about $25 \mathrm{msec}$ ) and that such an attenuated attentional capture is not strong enough to generate subsequent oculomotor capture. Even though the present data are consistent with the notion of a feature search and singleton detection mode (see Bacon \& Egeth, 1994), recent data by Theeuwes (in press) suggest that the notion of differential search modes may be incorrect, and he showed that the original results of Bacon and Egeth can be explained in terms of bottom-up salience signal.

The present findings are also related to results reported by Theeuwes and Burger (1998), who investigated whether a highly salient color singleton can be ignored during serial attentional search. They showed that one can ignore an irrelevant singleton only when both the target color and the distractor color are known and remain fixed over trials. When either the color of the target or the color of the distractor varied over trials, participants were not able to filter the distractor (see also Remington \& Folk, 1994, for a similar result). Even though Theeuwes and Burger investigated the predictability of only a single dimension (i.e., the color dimension) over trials, the present results are comparable in that in Experiment 1, in which the exact feature value of the tobe-searched dimension was not predictable over trials (i.e., it was unique in the shape dimension-either a circle between diamonds or a diamond between circles), there was a large interference effect and strong oculomotor capture. In the present Experiment 2 the interference effect was reduced (but still present) and there was no oculomotor capture. In line with Theeuwes and Burger and with Bacon and Egeth (1994), it appears that knowing the exact target feature to attend to and knowing the exact distractor feature to inhibit allows maximum top-down control and can prevent oculomotor capture by an irrelevant singleton. Note however that these findings do not imply that under these conditions there is no attentional capture. Indeed our Experiment 2 shows that the salient color singleton still caused an increase in manual RT, comparable to earlier experiments investigating attentional capture (e.g., Theeuwes, 1992). The salient singleton may have captured attention without generating a subsequent eye movement to the location of the color singleton. Similar conclusions were recently reached by Wu and Remington (2001), who showed that a reflexive shift of attention does not necessarily initiate the execution of a saccade. For example, in Wu and Remington's Experiment 4, a reliable color distractor effect on manual RT of about $10 \mathrm{msec}$ was found, whereas there was hardly any oculomotor capture.

Our Experiment 1 indicates that a static singleton can capture the eyes just as effectively (or even more effectively) as abrupt onsets do. This indicates that a distractor does not need to be a transient to capture the eyes, a finding at odds with Irwin et al.'s (2000) conclusions; they argued that only abrupt onsets (transients) are special in their ability to capture attention. Irwin et al. employed the oculomotor capture paradigm to examine the effect of a color singleton during for abrupt onsets and vice versa. Thus, in one condition participants searched for an abrupt onset while a color singleton distractor was present, and in another condition participants searched for a color singleton while an abrupt onset distractor was present. With this setup there was no evidence for oculomotor capture by color singletons. Only when the color singletons were used as targets in the preceding session was there an increase in manual RT, providing evidence for attentional capture. The results of our Experiment 1 (and those of Wu \& Remington, 2001) show that static color singletons can capture attention and the eyes (Experiment 1). In addition, even when maximum opportunity is provided for top-down control, we still found attentional capture (Experiment 2). Note that this cannot have been due to transfer from one session to the next (as in Irwin et al., 2000), because participants always performed a practice session before data collection started. Obviously, there are many differences between the present setup and that of Irwin et al. The most important difference is that in Irwin et al. there was always a abrupt onset (transient) present in the display. Since Theeuwes (1994, 1995) showed that abrupt onsets are always more salient than color singletons, it is not surprising that during an abrupt onset, the presence of a color singleton has no effect on attention or eye movement behavior. In the present experiments, we ensured that there were no luminance transients as targets or distractors and we used singleton distractors that were known to be more salient than the singleton targets. Obviously under these circumstances one does get attentional and oculomotor capture with static singletons.

Wu and Remington (2001) also claimed that color singletons can capture the eyes (their Experiment 2), yet they showed that these color singletons have to be accompanied by luminance change to generate saccades. Even though it is clear that transients are quite effective in their ability to generate reflexive saccades, our Experiment 1 shows that static singletons can be just as effective as onsets in producing oculomotor capture.

The question is, why was there strong oculomotor capture in Experiment 1 and hardly any in Experiment 2? There are two ways to explain these results. One way is to assume the earlier discussed search modes proposed by Bacon and Egeth (1994). In this view Experiment 1 generated strong attentional and oculomotor capture because observers were forced to use a singleton detection mode. Bacon and Egeth assumed that in this search mode 
observers cannot be selective and therefore irrelevant singletons interfere with search. Experiment 2 showed less attentional capture and no oculomotor capture because observers could choose to search for a feature, and, as argued by Bacon and Egeth in this feature search mode, top-down control is possible, preventing attentional capture by irrelevant singletons. This seems to be a adequate explanation of the present findings. It should be noted, however, that the evidence for the existence of these differential search modes may not be as strong as often is assumed (see Theeuwes, in press).

Another way to reconcile these findings is to assume that irrespective of whether the exact feature values of the target and singleton distractors are known (as in Experiment 1) or not known (as in Experiment 2), an irrelevant salient singleton always captures attention to its location (see Theeuwes, 1991a, 1992, 1994; Theeuwes et al., 2000; Theeuwes \& Godijn, 2001). When the exact stimulus features of target and distractor are known (as in Experiment 2), it is quite feasible that one would be able to disengage attention from the location of the salient singleton very quickly (see Theeuwes, Atchley, $\&$ Kramer, 2000). Disengagement of attention can be so fast because the feature value of the target (circle) and the feature value of the distractor (green) are known to the participant and never change throughout the whole experiment. As soon as attention is captured by the location of the irrelevant singleton, attentional processing reveals the identity of the singleton. Top-down knowledge that the target is circular and green and not square and red speeds up the disengagement process. When only the target and distractor dimensions are known, as in our Experiment 1, disengagement may be much slower. Indeed, in the "attentional version" of the present experiments, in which the exact feature values were not known, Theeuwes (1991a) found a much larger interference than he did in his 1992 study, in which the feature values of both target and distractor were known (110 msec in Theeuwes, 1991a, vs. $20 \mathrm{msec}$ in Theeuwes, 1992). Furthermore, if we assume that attention has to reside at the location of the irrelevant singleton for a minimum amount of time to trigger the programming and execution of the saccade to that location (see Godijn $\&$ Theeuwes, 2002b), then it is easy to understand why we found oculomotor capture in Experiment 1 but not in Experiment 2. Therefore, one can argue that an attentional set for a specific feature value defining both the target and the distractor, as in our Experiment 2, allowed such a fast disengagement of attention from the irrelevant location of the distractor that a saccade to that location was prevented.

It is important to note that the fixation durations on the irrelevant-singleton color distractor in Experiment 1 were brief (about $151 \mathrm{msec}$; see Figure 3), but not as brief as when onsets are used as distractors (about $90 \mathrm{msec}$; see Theeuwes et al., 1999, and Godijn \& Theeuwes, 2002b). This may be related to difficulty in disengaging (see Theeuwes, Godijn, \& Pratt, in press). Because of the reduced top-down control in Experiment 1, it may be dif- ficult to disengage not only attention from the irrelevant singleton but also the eyes. Obviously, this is rather speculative and there may be other reasons why fixation durations are much shorter when an abrupt onset is used as a distractor.

\section{REFERENCES}

BAcon, W. F., \& Egeth, H. E. (1994). Overriding stimulus-driven attention capture. Perception \& Psychophysics, 55, 485-496.

Breitmeyer, B. C., \& GANZ, L. (1976). Implications of sustained and transient channels for theories of visual pattern masking, saccadic suppression, and information processing. Psychological Review, 83, $1-36$.

Deubel, H., \& Schneider, W. X. (1996). Saccade target selection and object recognition: Evidence for a common attentional mechanism. Vision Research, 6, 1827-1837.

Egeth, H. E., \& Yantis, S. (1997). Visual attention: Control, representation and time course. Annual Review of Psychology, 48, 269297.

FindLEy, J. M. (1997). Saccade target selection during visual search. Vision Research, 37, 617-631.

Folk, C. L., \& Remington, R. [W.] (1998). Selectivity in distraction by irrelevant featural singletons: Evidence for two forms of attentional capture. Journal of Experimental Psychology: Human Perception \& Performance, 24, 847-858.

Folk, C. L., Remington, R. W., \& Johnston, J. C. (1992). Involuntary covert orienting is contingent on attentional control settings. Journal of Experimental Psychology: Human Perception \& Performance, 18, 1030-1044.

Folk, C. L., Remington, R. W., \& Wright, J. H. (1994). The structure of attentional control: Contingent attentional capture by apparent motion, abrupt onset, and color. Journal of Experimental Psychology: Human Perception \& Performance, 20, 317-329.

Godisn, R. \& Pratt, J. (2002). Endogenous saccades are preceded by shifts of visual attention: Evidence from cross-saccadic priming effects. Acta Psychologica, 110, 83-102.

Godisn, R. \& Theeuwes, J. (2001, November). Parallel programming of saccades requires the parallel allocation of attention. Paper presented at the annual meeting of the Psychonomic Society, Orlando, FL.

Godisn, R., \& Theeuwes, J. (2002a). Oculomotor capture and inhibition of return. Psychological Research, 66, 234-246.

Godis, R., \& TheEUwes, J. (2002b). Parallel programming of saccades: Evidence for a competitive integration model. Journal of Experimental Psychology: Human Perception \& Performance, 28, 1039-1054.

Hoffman, J. E., \& Subramaniam, B. (1995). The role of visual attention in saccadic eye movements. Perception \& Psychophysics, 57, 787-795.

Irwin, D. E., Colcombe, A. M., Kramer, A. F., \& Hahn, S. (2000). Attentional and oculomotor capture by onset luminance and color singletons. Vision Research, 40, 1443-1458.

IтTI, L., \& KoсH, C. (2000). A saliency-based search mechanism for overt and covert shifts of visual attention. Vision Research, 40, 1489-1506.

Koch, C., \& Ullman, S. (1985). Shifts in selective visual attention: Towards the underlying neural circuitry. Human Neurobiology, 4, 219 227.

Kramer, A. F., Hahn, S., Irwin, D. E., \& Theeuwes, J. (1999). Attentional capture and aging: Implications for visual search performance and oculomotor control. Psychology \& Aging, 14, 135-154.

Kramer, A. F., Hahn, S., Irwin, D. E., \& Theeuwes, J. (2000). Age difference in the control of looking behavior: Do you know where your eyes have been? Psychological Science, 11, 210-217.

Munoz, D. P., \& Istvan, P. J. (1998). Lateral inhibitory interactions in the intermediate layers of the monkey superior colliculus. Journal of Neurophysiology, 79, 1193-1209.

Nothdurft, H. C. (2000). Salience from feature contrast: Variations with texture density. Vision Research, 40, 3181-3200.

Rafal, R., Egly, R, \& RHodes, D. (1994). Effects of inhibition of return on voluntary and visually guided saccades. Canadian Journal of Experimental Psychology, 48, 284-300. 
Remington, R. W., \& Folk, C. L. (1994, November). Attentional capture can depend on search mode. Paper presented at the annual meeting of the Psychonomic Society, St. Louis.

Remington, R. W., Johnston, J. C., \& Yantis, S. (1992). Involuntary attentional capture by abrupt onsets. Perception \& Psychophysics, 51, 279-290.

Rizzolatti, G., Riggio, L., Dascola, I., \& Umiltà, C. (1987). Reorienting attention across the horizontal and vertical meridians: Evidence in favor of a premotor theory of attention. Neuropsychologia, 25, 31-40.

RuZ, M., \& LupIáÑEZ, J. (2002). Attentional capture and exogenous orienting: Upon their automaticity and sensitivity to endogenous control. Psicológica, 23, 283-309.

SAGI, D., \& JulESZ, B. (1985). Detection versus discrimination of visual orientation. Perception, 14, 619-628.

Salthouse, T. A., \& Ellis, C. L. (1980). Determinants of eye fixation duration. American Journal of Psychology, 93, 207-234.

Simons, D. J. (2000). Attentional capture and inattentional blindness. Trends in Cognitive Sciences, 4, 147-155.

Sparks, D. L., \& Hartwich-Young, R. (1989). The deep layers of the superior colliculus. Review of Oculomotor Research, 3, 213-255.

TAYLOR, T. L., \& KLEIN, R. M. (1998). On the causes and effects of inhibition of return. Psychonomic Bulletin \& Review, 5, 625-643.

Theeuwes, J. (1991a). Cross-dimensional perceptual selectivity. Perception \& Psychophysics, 50, 184-193.

Theeuwes, J. (1991b). Exogenous and endogenous control of attention: The effect of visual onsets and offsets. Perception \& Psychophysics, 49, 83-90.

Theeuwes, J. (1992). Perceptual selectivity for color and form. Perception \& Psychophysics, 51, 599-606.

Theeuwes, J. (1993). Visual selective attention: A theoretical analysis. Acta Psychologica, 83, 93-154.

Theeuwes, J. (1994). Stimulus-driven capture and attentional set: Selective search for color and visual abrupt onsets. Journal of Experimental Psychology: Human Perception \& Performance, 20, 799-806.

Theeuwes, J. (1995). Abrupt luminance change pops out; abrupt color change does not. Perception \& Psychophysics, 57, 637-644.

Theeuwes, J. (1996). Perceptual selectivity for color and form: On the nature of the interference effect. In A. F. Kramer, M. G. H. Coles, \& G. D. Logan (Eds.), Converging operations in the study of visual attention (pp. 297-314). Washington, DC: American Psychological Association.

Theeuwes, J. (in press). Top-down search strategies cannot override attentional capture. Psychonomic Bulletin \& Review.

Theeuwes, J., Atchley, P., \& Kramer, A. F. (2000). On the time course of top-down and bottom-up control of visual attention. In S. Monsell \& J. Driver (Eds.), Control of cognitive processes: Attention \& performance XVIII (pp. 104-124). Cambridge, MA: MIT Press.

Theeuwes, J., \& Burger, R. (1998). Attentional control during visual search: The effect of irrelevant singletons. Journal of Experimental Psychology: Human Perception \& Performance, 24, 1342-1353.

Theeuwes, J., \& Godisn, R. (2001). Attention and oculomotor capture. In C. Folk \& B. Gibson (Eds.), Attraction, distraction, and action: Multiple perspectives on attentional capture (pp. 121-150). Amsterdam: Elsevier.
Theeuwes, J., \& Godisn, R. (2002). Irrelevant singletons capture attention: Evidence from inhibition of return. Perception \& Psychophysics, 64, 764-770.

Theeuwes, J., Godisn, R., \& Pratt, J. (in press). A new estimation of the duration of attentional dwell time. Psychonomic Bulletin \& Review.

Theeuwes, J., Kramer, A. F., Hahn, S., \& Irwin, D. E. (1998). Our eyes do not always go where we want them to go: Capture of the eyes by new objects. Psychological Science, 9, 379-385.

Theeuwes, J., Kramer, A. F., Hahn, S., Irwin, D. E., \& Zelinsky, G. J. (1999). Influence of attentional capture on oculomotor control. Journal of Experimental Psychology: Human Perception \& Performance, 25, 1595-1608.

Trappenberg, T. P., Dorris, M. D., Munoz, D. P., \& Klein, R. M. (2001). A model of saccade initiation based on the competitive integration of exogenous and endogenous signals in the superior colliculus. Journal of Cognitive Neuroscience, 13, 256-271.

Wu, S.-C., \& Remington, R. W. (2001, November). Oculomotor capture is contingent on attentional control settings. Paper presented at the annual meeting of the Psychonomic Society, Orlando, FL.

YAnTIS, S. (1996). Attentional capture in vision. In A. F. Kramer, M. G. H. Coles, \& G. D. Logan (Eds.), Converging operations in the study of visual attention (pp. 45-76). Washington, DC: American Psychological Association.

YANTIS, S. (2000). Goal-directed and stimulus-driven determinants of attentional control. In S. Monsell \& J. Driver (Eds.), Control of cognitive processes: Attention \& performance XVIII (pp. 73-103). Cambridge, MA: MIT Press.

YANTIS, S., \& EGETh, H. E. (1999). On the distinction between visual salience and stimulus-driven attentional capture. Journal of Experimental Psychology: Human Perception \& Performance, 25, 661-676.

Yantis, S., \& Hillstrom, A. P. (1994). Stimulus-driven attentional capture: Evidence from equiluminant visual objects. Journal of Experimental Psychology: Human Perception \& Performance, 20, 95107.

YANTIS, S., \& JoNIDES, J. (1984). Abrupt visual onsets and selective attention: Evidence from selective search. Journal of Experimental Psychology: Human Perception \& Performance, 10, 601-621.

\section{NOTES}

1. There is evidence suggesting that abrupt onsets capture attention not because they are transients but because they signal the appearance of a new object (see Yantis \& Hillstrom, 1994).

2. In this study the color singleton was more salient than the shape singleton. This is not a general finding. As Theeuwes (1992) demonstrated, the salience of a color singleton can be reduced by making the color difference between target and nontargets smaller. In that case a shape singleton can be more salient than a color singleton.

(Manuscript received February 27, 2002; revision accepted for publication January 7, 2003.) 